\title{
Determinants of Necrotizing Enterocolitis Among Neonates in Referral Hospitals of East and West Gojjam Zones of Amhara Regional State, Northwest Ethiopia, 2020.
}

\author{
Wondim Derbew \\ Bahir Dar University \\ Amare Dagnew ( $\sim$ dagnewamare@gmail.com ) \\ Bahir Dar University \\ Frehiwot Girma \\ Bahir Dar University
}

\section{Research Article}

Keywords: case control, necrotizing enterocolitis, neonates and East and West Gojjam

Posted Date: March 1st, 2021

DOI: https://doi.org/10.21203/rs.3.rs-250519/v1

License: (c) (1) This work is licensed under a Creative Commons Attribution 4.0 International License.

Read Full License 
Determinants of necrotizing enterocolitis among neonates in referral hospitals of East and West Gojjam zones of Amhara regional state, Northwest, Ethiopia, 2020.

Amare Belachew Dagnew ${ }^{1}$, Wondim Derbew ${ }^{1}$, Friehiwot Girma ${ }^{1}$

1 Department of pediatrics and child health, College of Medicne and Health Sciences, Bahir Dar University, Ethiopia

Corresponding author: Amare Belachew; email:dagnewamare@gmail.com 


\section{ABSTRACT}

Background: Necrotizing enterocolitis is one of the most common serious surgical disorders of neonates. It is a life-threatening emergency of the gastrointestinal tract in the neonatal period that causes morbidity and mortality of newborns. Although the burden of the disease is high and it is the major cause for neonatal death, there is limited information's about identifying the determinant factors that may help to reduce the neonatal deaths. Thus this study aimed to identify the determinants of necrotizing enterocolitis among admitted neonates in neonatal intensive care unit at referral hospitals of East and West Gojjam Zones of Amhara regional state, Northwest Ethiopia, 2020.

Methods: Unmatched case control study design was conducted among 246 neonates (82 cases and 164 controls) in neonatal intensive care unit from February 24 to April 24, 2020. Data was collected through face to face interview and reviewing medical charts of the neonates. Data was entered into Epi data and exported to SPSS for analysis. Bivariable logistic regression analysis was used to identify the candidate variables at $\mathrm{p} \leq 0.25$ and multivariable logistic regression analysis was employed to identify significant determinants at $p$ value $<0.05$. Adjusted odds ratio with 95\% CI was used to show the strength of association between exposure and outcome variables.

Result: A total of 246 neonates were included in the study. Duration of rupture of membrane $>18$ hours $[\mathrm{AOR}=4.287 ; 95 \% \mathrm{CI}(2.157-8.518)]$, low birth weight $[\mathrm{AOR}=3.592 ; 95 \% \mathrm{CI}(1.742-$ 7.407)], neonatal sepsis $[\mathrm{AOR}=3.553 ; 95 \%$ CI (1.76-7.174)] and types of enteral feeding (formula milk only $[\mathrm{AOR}=3.604 ; 95 \% \mathrm{CI}(1.548-8.39)]$ and mixed milk $(\mathrm{AOR}=2.416 ; 95 \% \mathrm{CI}$ (1.103-5.290)]) were determinants for necrotizing enterocolitis. 
Conclusions: Duration of rupture of membrane, low birth weight, neonatal sepsis and enteral feedings (formula milk and mixed milk) were significantly associated with necrotizing enterocolitis. Encouraging exclusive breastfeeding, providing adequate care for low birth weight babies and using septic precautions to avoid neonatal sepsis are the strategies to prevent necrotizing enterocolitis.

Key words: case control, necrotizing enterocolitis, neonates and East and West Gojjam,.

\section{Introduction}

Necrotizing enterocolitis (NEC) is the most common serious surgical disorder (1). It is a lifethreatening emergency of the gastrointestinal tract in the newborn period that causes neonatal morbidity and mortality among newborns. It is characterized by various degrees of mucosal or transmural necrosis of the intestine $(1,2)$. The manifestations of necrotizing enterocolitis are abdominal distention, feeding intolerance, vomiting, blood in stool, abdominal wall erythema and systemic instability. Both clinical signs and radiographic findings are important to diagnose necrotizing enterocolitis. Plain abdominal radiographs are essential to make a diagnosis of NEC. The finding of pneumatosis intestinalis (air in the bowel wall) confirms the clinical suspicion of NEC (1-4).

$\mathrm{NEC}$ is the most common one of the gastrointestinal emergencies in premature infants in the neonatal intensive care unit (NICU) (5). Premature infants are accounts to $90 \%$ to develop NEC, while, full-term and near-term infants also develop the disease when they expose to risk factors $(5,6)$.

The exact pathogenesis of NEC remains unclear, but prematurity, enteral feeding, bacterial products, and intestinal ischemia have all been shown to cause activation of the inflammatory cascade. Awareness of the risk factors for NEC, early trophic feeding with breast milk and 
following the established feeding guidelines have been shown to reduce the incidence of NEC (7).

The first 28 days of life (neonatal period) is the most vulnerable time for a child's survival. Children face the highest risk of dying in their first month of life at a global rate of 18 deaths per 1,000 live births. Globally, an estimated 2.5 million newborns died in the first month of life, approximately 7,000 every day (8).

A study in China showed that the incidence of NEC was $2.50 \%$ and $4.53 \%$ in the low birth weight and very low birth weight infants, respectively (9). The mortality rates of very low birth weight infants and moderately low birth weight infants with confirmed NEC were $50.2 \%$ and $36.3 \%$, respectively. Another study in Canada stated that NEC affects $5-12 \%$ of very low birth weight babies and resulted $50 \%$ of fatal cases. The number of cases undergoing to surgery in this study was $20-40 \%$ of cases and leads health care cost more expensive (10).

World Health Organization report showed that among the sustainable development goal (SDG) regions, sub-Saharan Africa had the highest neonatal mortality rate in 2018 at 28 deaths per 1,000 live births, followed by Central and Southern Asia with 25 deaths per 1,000 live births. These findings suggest that focusing on the critical periods before and immediately following birth is essential to saving more newborn lives(8). NEC in Nigeria contributes to the commonest acquired and emergency surgical conditions that contributing for neonatal death of $11.8 \%(11)$. Another study in Cairo, Egypt, showed that $16.4 \%$ neonates were diagnosed with NEC and resulted $38.6 \%$ neonatal deaths (12).

According to the 2016 Ethiopian Demographic Health Survey (EDHS) report, the neonatal mortality rate was 29/1000 live births, which had no significant reduction from the 2011 EDHS report which was 37/1000 live births(13). About $4 \%$ neonates admiited in NICU with the case of 
NEC and the fatality of NEC was $46.2 \%$. It indicates that, it is a common fatal disease among neonatal morbidities (14). Identifying the risk factors and early initiation of therapy can significantly reduce the burden of neonatal death and illness related to NEC. The major causes of neonatal death in premature infants in Ethiopia are gestational age of 28-31 weeks,32-34 weeks and 35-36 weeks with NEC 2(22.2\%),5(55.6\%) and 2(22.2\%), respectively (15). This shows that, NEC is one of a contributing factor for neonatal mortality in Ethiopia.

There is a limited information regards NEC in the study area, Ethiopia as a whole and Africa. In addition, there are some contradicting or inconsistent findings on some factors for NEC, like prematurity, low birth weight and enteral feedings around the world. It gives a little attention although it is a very fatal case of neonates. Therefore, this study was aimed to identify determinants of NEC in neonatal intensive care unit.

\section{Methods}

\section{Study area and period}

This study was conducted in East and West Gojjam zone referral hospitals, Amhara Region, Ethiopia, from February 24 to April 24, 2020. There are three referral hospitals in these zones (Felege Hiwot compressive referral hospital (FHCRH), Tibebe Gion Specialized teaching hospital (TGSTH) and Debre Markos referral hospital (DMRH)). FHCRH and TGSTH are found in Bahir Dar city, the town of Amhara regional state in the Northern direction of 565 Kilometer

far from Addis Ababa (capital City of Ethiopia), whereas DMRH is found in Debre Markos, the town of East Gojjam Administrative Zone,299 Kilometers far from Addis Ababa. According to information obtained from administrative offices, FHCRH, TGSTH and DMRH have annual neonatal admission of more than 3500 (monthly 292), 1800 neonates and 2500 neonates, respectively. 


\section{Study design}

Institutional based unmatched case control study design was conducted among neonates admitted in NICU.

\section{Source population}

The source population was all admitted neonates in NICU from February 24 to April 24, 2020 at referral hospitals of East and West Gojjam Zones of Amhara regional state, Northwest Ethiopia.

\section{Study population}

The study population was all randomly selected neonates from February 24 to April 24, 2020 who fulfill the inclusion criteria for cases and controls among admitted neonates in NICU.

\section{Study units}

The study units were mothers and neonates on which the data was collected during data collection period.

\section{Inclusion and exclusion criteria}

\section{Inclusion criteria for cases}

All neonates who were admitted in the NICU of the three referral hospitals with clinical signs, radiographic finding of NEC and who being exclusively diagnosed with NEC by the attending physician at the time of data collection were included in the study.

\section{Inclusion criteria for controls}

All neonates who were admitted with other health problems to NICU during data collection period were included as controls. 


\section{Exclusion criteria for cases}

Neonates who were firstly diagnosed with NEC but recovered from NEC and developed other problems during data collection were excluded.

\section{Exclusion criteria for controls}

Neonates who were diagnosed with NEC and other health problems as the same time during data collection were not included as controls in the study.

\section{Variables of the study \\ Dependent variable}

Necrotizing enterocolitis

\section{Independent variables}

Socio-demographic variables: Maternal age, sex of the neonate, age of the neonate, residence, religion, maternal marital status, maternal occupation and educational status.

Maternal related variables: Prolonged rupture of membrane, Preeclampsia, ANC follow up, maternal diabetes mellitus, place of delivery and mode of delivery.

Neonatal related variables: Gestational age, birth weight, neonatal sepsis, neonatal asphyxia and multiple gestations.

Enteral feeding related variables: Breast milk, formula milk and mixed milk

\section{Operational definition}

Necrotizing enterocolitis(NEC): a surgical disorder and life-threatening emergency of the gastrointestinal tract in the neonatal period which has clinical signs of abdominal distention, abdominal wall erythema, feeding intolerance, vomiting, and blood in the stool $(1,2)$. 
Cases: neonates who presented with clinical signs and radiographic findings of NEC and who diagnosed by attending physician during data collection period.

Controls: neonates who were not diagnosed as NEC (do not fulfill clinical signs and radiographic findings of NEC) and who admitted with other health problems during data collection period.

Multiple gestations: a pregnancy with more than one fetus. It includes twins, triplets, quadruplets, or more(16).

Prolonged rupture of membrane: the time from membrane ruptured to delivery which is more than 18 hours(17).

Low birth weight: neonate's weight $<2500$ grams at birth.

Enteral feedings: Feedings that given to neonate though the mouth or through a tube that goes directly to the $\operatorname{stomach}(18)$.

Formula milk: artificial milk (manufactured milk) which is prepared by mixing the powder milk with water.

Mixed milk: the combination of breast milk and formula milk

\section{Sample size determination}

The sample size was calculated by using Epi-Info version 7 software by considering the proportion of mixed milk of cases (31\%), the proportion of controls exposed (15\%), ratio $(1: 2)$, $80 \%$ power , and $95 \%$ confidence level [36-38]. Then, it gives a total estimated sample size of 234, (78 cases and 156 controls). By adding a 5\% non-response rate, a total of 246 ( 82 cases and 164 controls) participants were included in this study. 


\section{Sampling procedures and techniques}

Proportionate allocation was made for each referral hospitals to select cases and controls.

Cases and two corresponding controls were selected randomly (lottery method) among those neonates admitted in the NICU of the three referral hospitals. One hundred eleven neonates (37 cases 74 controls), 57 neonates (19 cases 38 controls) and 78 neonates (26 cases 52 controls) were selected from FHCRH, TGTSH and DMRH, respectively.

\section{Data Collection procedures and instruments}

An interviewed structured questionnaire [36-38] was used to collect the data. Six BSc nurses collected the data. Medical cards and feeding charts of the case and control groups were used in addition to face to face interview. Mothers were interviewed about their socio-demographic characteristics and maternal factors where as neonatal and enteral feeding factors were collected from medical cards.

\section{Data quality control}

Training was given for data collectors and supervisors. A pretest was conducted in Addis Alem hospital which is found in Bahir Dar city by taking $5 \%$ of the sample size before actual data collection to check consistency and any ambiguous of the language. A clear explanation about the purpose and objective of the study was provided for the respondents at the beginning of the interview. Close supervision was carried out by the principal investigator and supervisors during data collection period.

\section{Data processing and analysis}

The data was coded and entered into EPI data statistical software version 3.1, then exported to SPSS statistical software version 20 for data cleaning and analysis. Bivariable analysis was done to observe the candidate variables at $\mathrm{p}-$ value $\leq 0.25$ and then multivariable logistic regression 
analysis was employed to identify the independent variables that associated with NEC at p-value $<0.05$. Adjusted odds ratio (AOR) with $95 \%$ CI was used to show the strength of association between exposure and outcome variables. Frequency distribution tables and statistical graphs were used to describe some variables. 


\section{RESULTS}

\section{Socio-demographic characteristics}

This study was intended to identify determinants of necrotizing enterocolitis among neonates admitted in NICU at referral hospitals of East and West Gojjam Zones. A total of 246 neonates (82 cases and 164 controls) who were admitted in NICU with their mothers were included with the overall response rate of $100 \%$. According to this study, the median age of the mothers of cases and controls was 26.5 and $27(25-34)$ years, respectively. More than half of the participants $47(57.3 \%)$ cases and $85(51.8 \%)$ controls were from urban areas. Fifty six $(68.3 \%)$ cases and 86(52.4\%) controls were males (Table-1).

Table 1: Socio demographic characteristics of neonates and their mothers at referral hospitals of East and West Gojjam zones of Amhara regional state, Northwest Ethiopia, 2020(n=246).

\begin{tabular}{llll}
\hline Variable & category & Cases(n=82) & Controls( $(\mathrm{N})$ \\
& & № $(\%)$ & \\
& & & \\
Mother's age & $15-24$ & $29(35.4)$ & $52(31.7)$ \\
& $25-34$ & $37(45.1)$ & $84(51.2)$ \\
Marital status & $35-49$ & $16(19.5)$ & $28(17.1)$ \\
& Married & $72(87.8)$ & $136(82.9)$ \\
& Widowed & $5(6.1)$ & $15(9.1)$ \\
Deligion & Divorced & $5(6.1)$ & $13(7.9)$ \\
& Orthodox & $52(63.4)$ & $99(60.4)$ \\
& Muslim & $21(25.6)$ & $55(33,5)$ \\
& Other & $9(11.0)$ & $10(6.1)$
\end{tabular}




\begin{tabular}{|c|c|c|c|}
\hline \multirow[t]{2}{*}{ Residence } & Urban & $47(57.3)$ & $85(51.8)$ \\
\hline & Rural & $35(42.7)$ & $79(48.2)$ \\
\hline \multirow{4}{*}{$\begin{array}{l}\text { Maternal educational } \\
\text { status }\end{array}$} & Cannot read and write & $30(36.6)$ & $50(30.5)$ \\
\hline & Primary & $17(20.7)$ & $39(23.8)$ \\
\hline & Secondary & $15(18.3)$ & $49(29.9)$ \\
\hline & College and higher & $20(24.4)$ & $26(15.9)$ \\
\hline \multirow[t]{5}{*}{ Occupation } & Housewife & $38(46.3)$ & $84(51.2)$ \\
\hline & Government employee & $17(20.7)$ & $23(14.0)$ \\
\hline & Merchant & $16(19.5)$ & $37(22.6)$ \\
\hline & Private organization & $5(6.1)$ & $6(3.7)$ \\
\hline & Other & $6(7.3)$ & $14(8.5)$ \\
\hline \multirow[t]{4}{*}{ Monthly income } & $<500$ & $19(23.2)$ & $47(28.7)$ \\
\hline & $500-1000$ & $5(6.1)$ & $22(13.4)$ \\
\hline & $1000-1500$ & $10(12.2)$ & $14(8.5)$ \\
\hline & $>1500$ & $48(58.5)$ & $81(49.4)$ \\
\hline \multirow[t]{2}{*}{ Neonate's sex } & Male & $56(68.3)$ & $86(52.4)$ \\
\hline & Female & $26(31.7)$ & $78(47.6)$ \\
\hline \multirow[t]{4}{*}{ Neonate's age } & 1-7 days & $42(51.2)$ & $92(56.1)$ \\
\hline & 7-14 days & $22(26.8)$ & $39(23.8)$ \\
\hline & 14-21 days & $9(11.0)$ & $21(12.8)$ \\
\hline & 21-28 days & $9(11.0)$ & $12(7.3)$ \\
\hline
\end{tabular}

Key: Other=catholic, protestant, farmer, daily labor 


\section{Maternal related factors for necrotizing enterocolitis}

The majority of mothers $51(62.2 \%)$ of cases and $117(71.3 \%)$ of controls had got ANC follow up. Thirty seven (41.5\%) mothers of cases and 49(29.9\%) of controls had history of preeclampsia during their pregnancy. About 42(51.2\%) cases and 137(83.5\%) controls were duration of rupture of membrane $\leq 18$ hours whereas $40(48.8 \%)$ cases and $27(16.5 \%)$ controls were $>18$ hours (Table-2).

Table 2: Description of maternal related factors of NEC among neonates admitted in NICU at referral hospitals of East and West Gojjam Zones of Amhara regional state, Northwest Ethiopia, 2020.

\begin{tabular}{|c|c|c|c|}
\hline \multirow[t]{2}{*}{ Variable } & \multirow[t]{2}{*}{ category } & Cases $(n=82)$ & Controls $(n=164)$ \\
\hline & & № $(\%)$ & No (\%) \\
\hline \multirow[t]{2}{*}{ ANC follow up } & Yes & $51(62.2)$ & $117(71.3)$ \\
\hline & No & $31(37.8)$ & $47(28.7)$ \\
\hline \multirow[t]{2}{*}{ Number of ANC visit } & $<3$ & $28(54.9)$ & $72(61.5)$ \\
\hline & $\geq 3$ & $23(45.1)$ & $45(38.5)$ \\
\hline \multirow[t]{2}{*}{ preeclampsia } & Yes & $34(41.5)$ & 49(29.9) \\
\hline & No & $48(58.5)$ & $115(70.1)$ \\
\hline \multirow[t]{2}{*}{ Maternal DM } & Yes & $6(7.3)$ & $21(12.8)$ \\
\hline & No & $76(92.7)$ & $143(87.2)$ \\
\hline \multirow{2}{*}{$\begin{array}{l}\text { Duration of rupture of } \\
\text { membrane }\end{array}$} & $\leq 18 \mathrm{hrs}$ & $42(51.2)$ & $137(83.5)$ \\
\hline & $>18 \mathrm{hrs}$ & $40(48.8)$ & $27(16.5)$ \\
\hline \multirow[t]{3}{*}{ Place of delivery } & Home & $8(9.8)$ & $16(9.8)$ \\
\hline & Health center & $52(63.4)$ & $74(45.1)$ \\
\hline & hospital & $22(26.8)$ & 74(45.) \\
\hline
\end{tabular}


Regarding to mode of delivery, majority of mothers $47(57.3 \%)$ of cases and three fourth 123(75.0\%) of controls had spontaneous vaginal delivery (Figure-1).

\section{Modes of delivery}

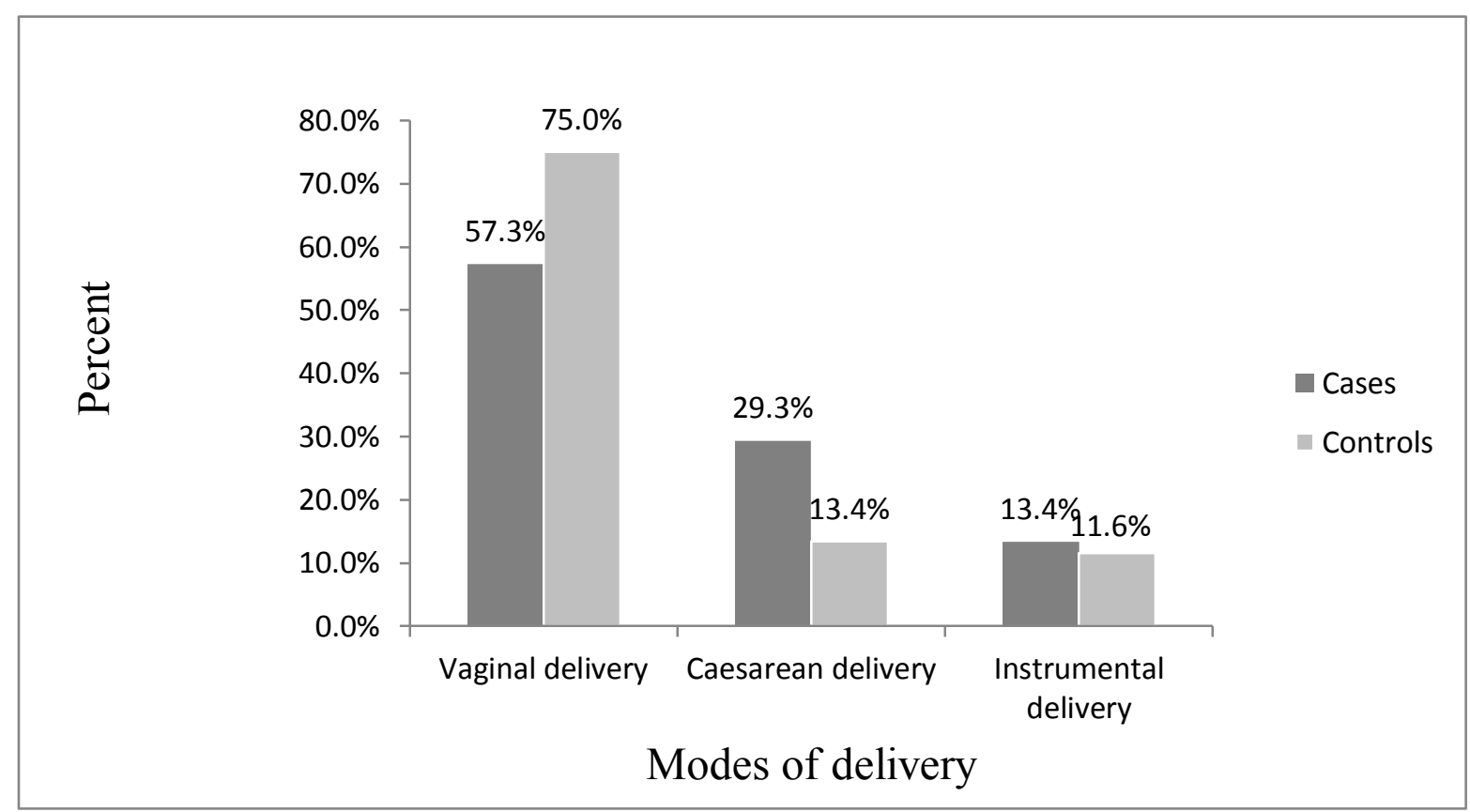

Figure 1: Mode of delivery in cases and controls for the study of determinants of necrotizing enterocolitis among neonates admitted in NICU at referral hospitals of East and West Gojjam Zones of Amhara regional state, Northwest Ethiopia.

\section{Neonatal related factors for necrotizing enterocolitis}

Sixty seven $(81.7 \%)$ cases and $80(48.8 \%)$ controls were preterm neonates $(<37$ weeks). The median gestational age of cases and controls was 35 weeks and 37 weeks as well as the median of birth weight of cases and controls was 2000 and 2585 grams, respectively. Sixty three (76.8\%) cases and 81(49.4\%) of controls were low birth weight (Table-3). 
Table 3: Description of neonatal related factors of NEC among neonates admitted in NICU at referral hospitals of East and West Gojjam Zones of Amhara regional state, Northwest Ethiopia, 2020.

\begin{tabular}{|c|c|c|c|}
\hline Variable & category & Cases(n=82) & Controls $(\mathrm{n}=164)$ \\
\hline & & № $(\%)$ & No $(\%)$ \\
\hline \multirow[t]{2}{*}{ Gestational age } & $<37$ weeks & $67(81.7)$ & $80(48.8)$ \\
\hline & $37-42$ weeks & $15(18.3)$ & $84(51.2)$ \\
\hline \multirow[t]{2}{*}{ Birth weight } & $<2500$ grams & $63(76.8)$ & $81(49.4)$ \\
\hline & $\geq 2500$ grams & $19(23.2)$ & $83(50.6)$ \\
\hline \multirow[t]{2}{*}{ Multiple gestation } & Yes & $16(19.5)$ & $32(19.5)$ \\
\hline & No & $66(80.5)$ & $132(80.5)$ \\
\hline \multirow[t]{2}{*}{ Type of multiple gestation } & twin & $15((93.8)$ & $32(100)$ \\
\hline & triplet & $1(6.2)$ & $0(0)$ \\
\hline \multirow[t]{2}{*}{ Neonatal sepsis } & Yes & $67(81.7)$ & $77(47.0)$ \\
\hline & No & $15(18.3)$ & $87(53.0)$ \\
\hline \multirow[t]{2}{*}{ Neonatal HIV status } & Exposed & $9(11.0)$ & $13(7.9)$ \\
\hline & Non-exposed & $73(89.0)$ & $151(92.1)$ \\
\hline \multirow[t]{2}{*}{ Neonatal asphyxia } & Yes & $23(28.0)$ & $56(34.1)$ \\
\hline & No & $59(72.0)$ & $108(65.9)$ \\
\hline
\end{tabular}




\section{Types of enteral feeding}

Regarding to types of enteral feeding, majority $37(45.1 \%)$ of cases and $113(68.9 \%)$ controls were fed breast milk only, 21(25.6\%) cases and 24(14.6\%) controls were fed formula milk only and 24(29.3\%) cases and 27(16.5\%) controls were fed mixed milk (Figure-2).

\section{Types of enteral feeding}

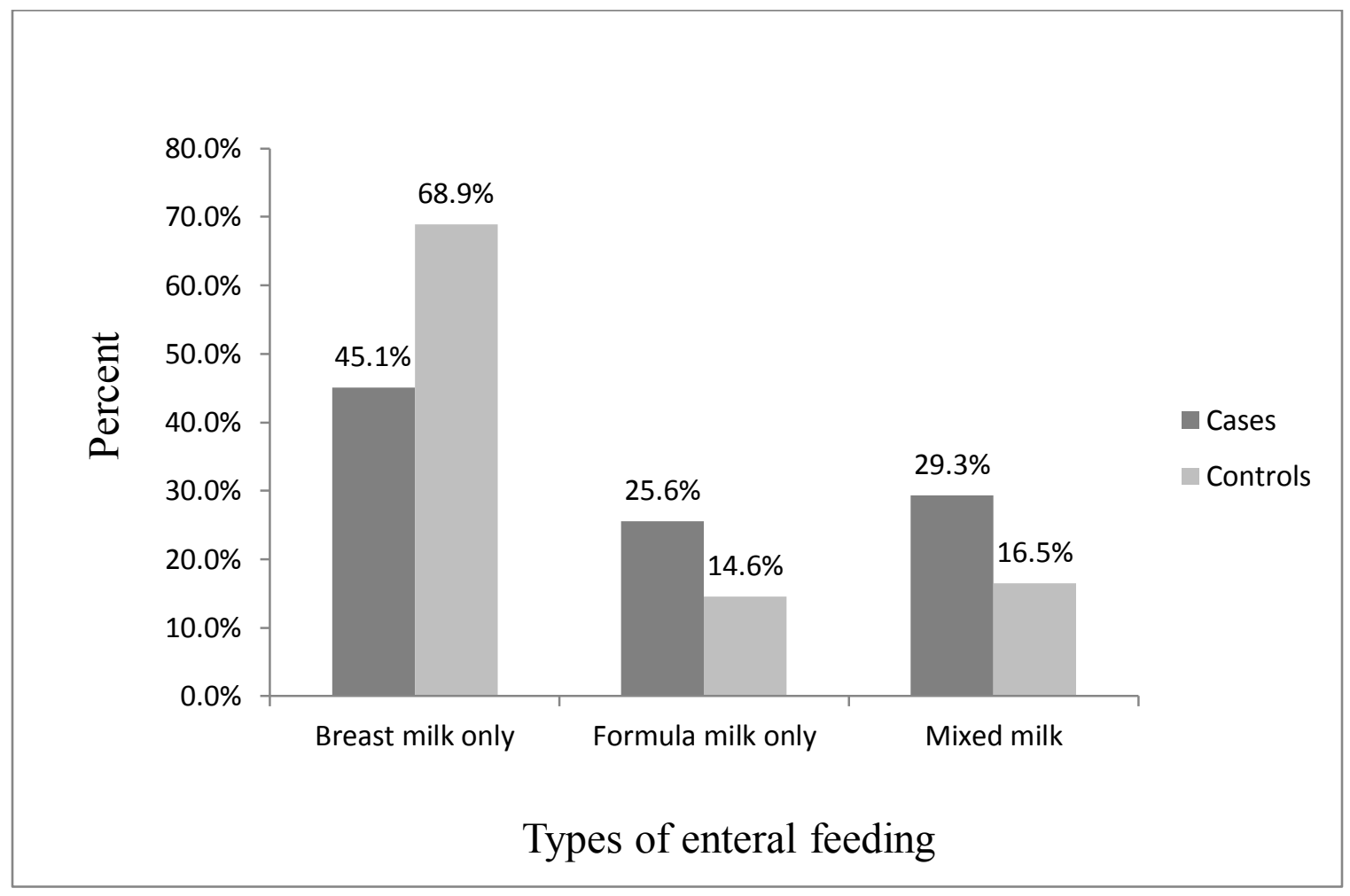

Figure2: Types of enteral feeding for the study of determinants of necrotizing enterocolitis among neonates admitted in NICU at referral hospitals of East and West Gojjam Zones of Amhara regional state, Northwest Ethiopia, 2020. 


\section{Bivariable and multivariable logistic regression analysis of determinants associated with necrotizing enterocolitis}

Mother's educational status, occupation, monthly income, neonatal sex, ANC follows up, preeclampsia, maternal DM, duration of rupture of membrane, place of delivery, mode of delivery, gestational age, birth weight, neonatal sepsis and types of enteral feeding had shown significant association during the bivariable logistic regression at $\mathrm{P}$-value $\leq 0.25$. Of these, duration of rupture of membrane, birth weight, neonatal sepsis and types of enteral feeding were found to be significant association with NEC by multivariable logistic regression at p-value $<0.05$.

Hence, duration of rupture of membrane was significantly associated with NEC. Mothers with duration of rupture of membrane $>18$ hours were 4.28 times more likely to have neonates with NEC compared to mothers with duration of rupture of membrane $\leq 18$ hours $[\mathrm{AOR}=4.287$; 95\%CI (2.157-8.518), $\mathrm{p}<0.001]$.

Birth weight was significantly associated with NEC. The odds of having NEC was 3.59 times higher among neonates who had low birth weight ( $<2500$ grams) compared to those neonates who had normal birth weight $(\geq 2500$ grams $)[\mathrm{AOR}=3.592 ; 95 \%$ CI $(1.742-7.407), \mathrm{p}$ $=0.001)]$. Similarly, neonatal sepsis was significantly associated with NEC. Neonates with sepsis were 3.55 times more likely to have NEC compared to those neonates without sepsis $[(\mathrm{AOR}=3.553 ; 95 \% \mathrm{CI}(1.76-7.174), \mathrm{p}<0.001]$

In addition to these, enteral feeding was significantly associated with NEC. Specifically, neonates who fed formula milk only were 3.6 times more likely to have NEC compared to those who fed breast milk only [(AOR=3.604; 95\% CI (1.548-8.390), $\mathrm{p}=0.003]$. As well as, neonates 
who fed mixed milk were 2.4 times more likely to have NEC compared to those neonates fed breast milk only [(AOR=2.416; 95\% CI (1.103-5.290), $\mathrm{p}=0.027]$ (Table-4)

Table 4: Bivariable and multivariable logistic regression analysis of factors associated with NEC among neonates admitted in NICU at referral hospitals of East and West Gojjam Zones of Amhara regional state, Northwest Ethiopia, 2020.

\begin{tabular}{|c|c|c|c|c|c|}
\hline \multirow{3}{*}{ Variable } & \multirow{3}{*}{ category } & Cases & Controls & \multirow{3}{*}{$\operatorname{COR}(95 \% \mathrm{CI})$} & \multirow{3}{*}{$\mathrm{AOR}(95 \% \mathrm{CI}$} \\
\hline & & $(n=82)$ & $(n=164)$ & & \\
\hline & & № $(\%)$ & № (\%) & & \\
\hline \multirow{4}{*}{$\begin{array}{l}\text { Mother's } \\
\text { educational } \\
\text { status }\end{array}$} & Cannot read and write & $30(36.6)$ & $50(30.5)$ & $0.78(0.373-1.632)$ & $1.008(0.396-2.565)$ \\
\hline & Primary & $17(20.7)$ & $39(23.8)$ & $0.567(0.251-1.280)$ & $0.630(0.233-1.702)$ \\
\hline & Secondary & $15(18.3)$ & $49(29.9)$ & $0.398(0.175-0.905) *$ & $0.380(0.137-1.054)$ \\
\hline & College and higher & $20(24.4)$ & $26(15.9)$ & 1 & 1 \\
\hline \multirow[t]{5}{*}{ Occupation } & Housewife & $38(46.3)$ & $84(51.2)$ & 1 & 1 \\
\hline & Government employee & $17(20.7)$ & $23(14.0)$ & $1.634(0.784-3.408) *$ & $1.948(0.559-6.789)$ \\
\hline & merchant & $16(19.5)$ & $37(22.6)$ & $0.956(0.474-1.926)$ & $1.206(0.191-7.607)$ \\
\hline & Private organization & $5(6.1)$ & $6(3.7)$ & $1.842(0.529-6.411)$ & $1.273(0.264-6.153)$ \\
\hline & Other & $6(7.3)$ & $14(8.5)$ & $0.947(3.38-2.654)$ & $1.514(0.185-12.355)$ \\
\hline \multirow{4}{*}{$\begin{array}{l}\text { Monthly } \\
\text { income }\end{array}$} & $<500$ & $19(23.2)$ & $47(28.7)$ & $0.682(0.359-1.296)^{*}$ & $0.67(0.264-1.704)$ \\
\hline & $500-1000$ & $5(6.1)$ & $22(13.4)$ & $0.384(0.136-1.079)^{*}$ & $0.309(0.08-1.187)$ \\
\hline & $1000-1500$ & $10(12.2)$ & $14(8.5)$ & $1.205(0.497-2.925)$ & $1.433(0.461-4.450)$ \\
\hline & $>1500$ & $48(58.5)$ & $81(49.4)$ & 1 & 1 \\
\hline \multirow[t]{2}{*}{ Neonate's sex } & Male & $56(68.3)$ & $86(52.4)$ & $1.953(1.119-3.410)^{*}$ & $1.607(0.827-3.122)$ \\
\hline & Female & $26(31.7)$ & $78(47.6)$ & 1 & 1 \\
\hline ANC follow up & Yes & $51(62.2)$ & $117(71.3)$ & 1 & 1 \\
\hline
\end{tabular}




$$
\text { No }
$$

$31(37.8)$

preeclampsia $\quad$ Yes
No

Maternal DM Yes

No

Duration of $\leq 18 \mathrm{hrs}$

rupture of

membrane

$>18$ hrs

Place of

Home

delivery

Health center

Hospital

Mode of

delivery

Vaginal delivery

Cesarean section

Instrumental delivery

Gestational age $\quad<37$ weeks

37-42 weeks

Birth weight $\quad<2500$ grams

$\geq 2500$ grams

Neonatal sepsis Yes

No

$34(41.5)$

$$
6(7.3)
$$

$$
8(9.8)
$$

$52(63.4)$

$47(57.3)$

$11(13.4)$

$63(76.8)$

$19(23.2)$
48(58.5)

49(29.9)

115(70.1)

$1.66(0.957-2.887) *$

1

21(12.8)

$0.538(0.208-1.389)^{*}$

$0.4(0.122-1.316)$

76(92.7) 143(87.2)

42(51.2) 137(83.5)

40(48.8)

27(16.5)

4.832(2.657-8.789)*

$4.287(2.157-8.518)^{* *}$

$16(9.8)$

1.682(0.636-4.450)

$1.283(0.341-4.830)$

22(26.8)

$74(45.1)$

$2.364(1.306-4.279)^{*}$

$1.262(0.55-2.896)$

$74(45.1)$

24(29.3)

123(75.0)

22(13.4)

$2.855(1.462-5.574)^{*}$

$2.305(1.007-5.275)$

19(11.6)

$1.515(0.671-3.423)$

$0.881(0.35-2.216)$

$\begin{array}{cccc}67(81.7) & 80(48.8) & 4.69(2.478-8.878)^{*} & 1.881(0.538-6.580) \\ 15(18.3) & 84(51.2) & 1 & 1\end{array}$

81(49.4)

$3.398(1.87-6.175) *$

$3.592(1.742-7.407)^{* *}$

83(50.6)

$67(81.7) \quad 77(47.0)$

$5.047(2.666-9.555)^{*}$

$3.553(1.76-7.174)^{* *}$

$15(18.3) \quad 87(53.0)$

1
1 


\begin{tabular}{llcccc}
$\begin{array}{c}\text { Types of enteral } \\
\text { feeding }\end{array}$ & Breast milk & $37(45.1)$ & $113(68.9)$ & 1 & 1 \\
& & & & & \\
& Formula milk & $21(25.6)$ & $24(14.6)$ & $2.672(1.336-5.346)^{*}$ & $3.604(1.548-8.390)^{* *}$ \\
& & & & \\
& & & & & \\
& Mixed milk & $24(29.3)$ & $27(16.5)$ & $2.715(1.398-5.271)^{*}$ & $2.416(1.03-5.290)^{* *}$ \\
\hline
\end{tabular}

Key; * = candidate variables at $\mathrm{p} \leq 0.25$ in bivariable logistic regression, ** significant variables at $\mathrm{p}<0.05$ in multivariable logistic regression, other $=$ farmer, daily labor and $1=$ reference category 


\section{DISCUSSION}

Neonatal enteroclitis is the major problems of neonatal deaths. Thus, identifying the determinant factors is crucial to enhance the health of neonates.

Duration of rupture of membrane ( $>18$ hours) was significantly associated with NEC. Neonates born from mothers with duration of rupture of membrane $>18$ hours were more likely to suffer from NEC as compared to those neonates born from mothers with duration of rupture of membrane $\leq 18$ hours. This is consistent with studies conducted in USA(19) and Sweden (20). The possible explanation might be due to birth canal is colonized with aerobic and anaerobic pathogens (from amniotic fluid) that might also cause colonization of the neonate's intestine at birth when duration of labor is greater than eighteen hours.

Birth weight was significantly associated with NEC. Neonates with low birth weight $(<2500$ grams $)$ were more likely to have NEC as compared to neonates with normal birth weight ( $\geq 2500$ grams). This finding is in line with findings of studies conducted in Sweden (20), Malaysia (21) and South Africa (22). This is due to the fact that low birth weight is developed due to prematurity and maternal complication like hypertension that present pre-conception or antepartum (2). Another neonatal variable in this study which was found to be significantly associated with NEC was neonatal sepsis. The present study revealed that neonates with sepsis were more likely to have NEC compared to those neonates without sepsis. This finding is also consistent with studies conducted in Sweden(20), USA(19) and China(23). The possible reason to this is; in the fact that neonate's immune system is not yet fully developed and they are susceptive to infection during invasive procedures in the NICU and also when a neonate has septicemia (sepsis), bacteria reproduce in the blood and produce toxins. The immature intestinal tract is exposed to these factors, resulting in a large number of cytokines that mediate an 
inflammatory cascade. NEC is thought to be precipitated by an inflammatory cascade that causes cytokine release and damage to the immature intestine $(1,2)$

In addition to these, multivariable logistic regression modeling demonstrated that enteral feeding was significantly associated with NEC. This study showed that specifically neonates who fed formula milk only were more likely to have NEC compared to those who fed breast milk only and neonates who fed mixed milk were also more likely to have NEC compared to those neonates fed breast milk only. Similar finding was reported from studies conducted in England (24), Scotland (25), Netherlands (26) and South Africa (22) also revealed that neonates who fed formula milk only were more likely to have NEC compared to those who fed breast milk only. The possible reasons might be immaturity of bowel function of the neonates for digestion of formula milk(1). In addition to this, formula milk lacks immune protective factors that available in breast milk and it might contain food additives(5). Besides, feeding equipment that used to prepare the milk might always not be thoroughly cleaned before and after used.

\section{Conclusion and recommendation}

Duration of rupture of membrane ( $>18$ hours), low birth weight $(<1500$ grams), neonatal sepsis and enteral feedings (formula milk and mixed milk) were significantly associated with NEC. Thus, using aseptic precautions for low birth weight, encouraging exclusive breastfeeding, and avoiding delay of labor are recommended in order to prevent neonatal enterocolitis.

\section{Acronyms and abbreviations}

ANC: Antenatal care;

AOR: Adjusted odds ratio;

CI: Confidence interval; COR: 
Crude odds ratio;DMRH:

Debre Markos referral hospital;

EDHS:Ethiopian demographic and health survey;

FHCSRH: Felege Hiwot Compressive specialized referral hospital;

NEC; necrotizing enterocolitis;

NICU: Neonatal intensive care unit

TGSTH:Tibebe Ghion specialized teaching hospital 


\section{Declarations}

\section{Ethics approval and consent to participate}

Ethical clearance was obtained from Ethical Review Committee (ERC) of Bahir Dar University College of medicine and health sciences. Informed consent was obtained from all study participants (mothers) or parents for those have age less than 18 years-old. Written consent was obtained from participants after they were informed about the objectives, expected outcomes, benefits and the risks associated with it. Confidentiality and privacy of every respondent's information were ensured. All methods were performed in accordance with the relevant guidelines and regulations

\section{Consent for publication}

Not applicable

\section{Funding}

Not applicable

\section{Availability of the data and materials}

The datasets generated during and analyzed during the current study are not publicly available due to confidentiality of the participants' information but are available from the corresponding author on reasonable request.

\section{Competing interests}

The authors declare that they have no any competing interests.

\section{Acknowledgment}


Authors acknowledged the data collectors, supervisors, respondents and administrators of each referral hospitals for their contribution in this study completion.

\section{Authors' contributions}

Amare Belachew Dagnew and Wondim Derbew: designed the study, gave training for the data collectors, supervised the data collectors, analyzed and interpreted the result and prepared the manuscript. Frehiwot Girma had contribution in the data analysis, data interpretation and drafting of the manuscript. All authors read and approved the final manuscript.

\section{REFERENCES}

1. Federal Ministry of health of Ethiopia. Neonatal intesive care unit training participants' manual. 2014:55.

2. Robert M. Kliegman. Nelson textbook of pediatrics. 2018;2:1173.

3. Young Ah Youn E-KK, Kim: aSY. Necrotizing Enterocolitis among Very-Low-BirthWeight Infants

in Korea. 2015.

4. William_Hay ML,_Robin_Deterding,_Mark_A. current diagnosis and treatments of pediatrics 24 th edition 41 .

5. Ms Sheila M. Gephart R, BSN, Dr Jacqueline M,et al,. Necrotizing enterocoltis risk:Virginia. 2013.

6. Gephart MSM, McGrath JM, Effken JA, Halpern MD. Necrotizing enterocolitis risk: state of the science. Advances in Neonatal Care. 2012;12(2):77.

7. Choi YY. Necrotizing enterocolitis in newborns: update in pathophysiology and newly emerging therapeutic strategies. Korean journal of pediatrics. 2014;57(12):505. 
8. UNICEF W, World Bank group,United Nations,. Levels \& Trends in child mortality report. 2019.

9. Qian T, Zhang R, Zhu L, Shi P, Yang J, Yang C-y, et al. Necrotizing enterocolitis in low birth weight infants in China: mortality risk factors expressed by birth weight categories. Pediatrics \& Neonatology. 2017;58(6):509-15.

10. Shulhan J, Dicken B, Hartling L, Larsen BM. Current knowledge of necrotizing enterocolitis in preterm infants and the impact of different types of enteral nutrition products. Advances in nutrition. 2017;8(1):80-91.

11. Ugwu RO, Okoro PE. Pattern, outcome and challenges of neonatal surgical cases in a tertiary teaching hospital. African Journal of Paediatric Surgery. 2013;10(3):226.

12. Abdel Ghany EA, Ali AA. Empirical antibiotic treatment and the risk of necrotizing enterocolitis and death in very low birth weight neonates. Annals of Saudi medicine. 2012;32(5):521-6.

13. ICF: CSACEa. Ethiopia Demographic and Health Survey 2016. Addis Ababa, Ethiopia. 2016.

14. Kokeb M, Desta T. Institution Based prospective cross-sectional study on patterns of neonatal morbidity at Gondar University Hospital Neonatal Unit, North-West Ethiopia. Ethiopian journal of health sciences. 2016;26(1):73-9.

15. Muhe LM, McClure EM, Nigussie AK, Mekasha A, Worku B, Worku A, et al. Major causes of death in preterm infants in selected hospitals in Ethiopia (SIP): a prospective, crosssectional, observational study. The Lancet Global Health. 2019;7(8):e1130-e8.

16. https://www.sciencedirect.com/topics/medicine-and-dentistry/multiple-gestation. multiple-gestation 2019. 
17. Richard A. Polin WWF. Fetal and Neonatal Physiology. 2019.

18. https://www.healthline.com/health/enteral-feeding. enteral feeding. Feb 6,2020.

19. Drenckpohl D, Knaub L, Schneider C, McConnell C, Wang H, Macwan K. Risk factors that may predispose premature infants to increased incidence of necrotizing enterocolitis. ICAN: Infant, Child, \& Adolescent Nutrition. 2010;2(1):37-44.

20. Ahle M, Drott P, Elfvin A, Andersson RE. Maternal, fetal and perinatal factors associated with necrotizing enterocolitis in Sweden. A national case-control study. PloS one. 2018;13(3):e0194352.

21. Boo N-Y, Cheah G. Risk factors associated with necrotising enterocolitis in very low birth weight infants in Malaysian neonatal intensive care units. Singapore medical journal. 2012;53(12):826.

22. Angura P. Necrotising enterocolits in premature infants at Chris Hani Baragwanath hospital. What are the associated factors? 2014.

23. Zhang L-p, Lei X-p, Luo L-j, Dong W-b. Risk factors for necrotizing enterocolitis in very preterm infants: a case-control study in southwest China. The Journal of Maternal-Fetal \& Neonatal Medicine. 2019;32(6):896-901.

24. Lucas A, Cole TJ. Breast milk and neonatal necrotising enterocolitis. The Lancet. 1990;336(8730-8731):1519-23.

25. McGuire W, Anthony M. Donor human milk versus formula for preventing necrotising enterocolitis in preterm infants: systematic review. Archives of Disease in Childhood-Fetal and Neonatal Edition. 2003;88(1):F11-F4. 
26. Berkhout DJ, Klaassen P, Niemarkt HJ, de Boode WP, Cossey V, van Goudoever JB, et al. Risk factors for necrotizing enterocolitis: a prospective multicenter case-control study. Neonatology. 2018;114:277-84.

Supplementary file 1: The English version of the questionnaire along with the Amharic version that used to collect the data. 
Figures

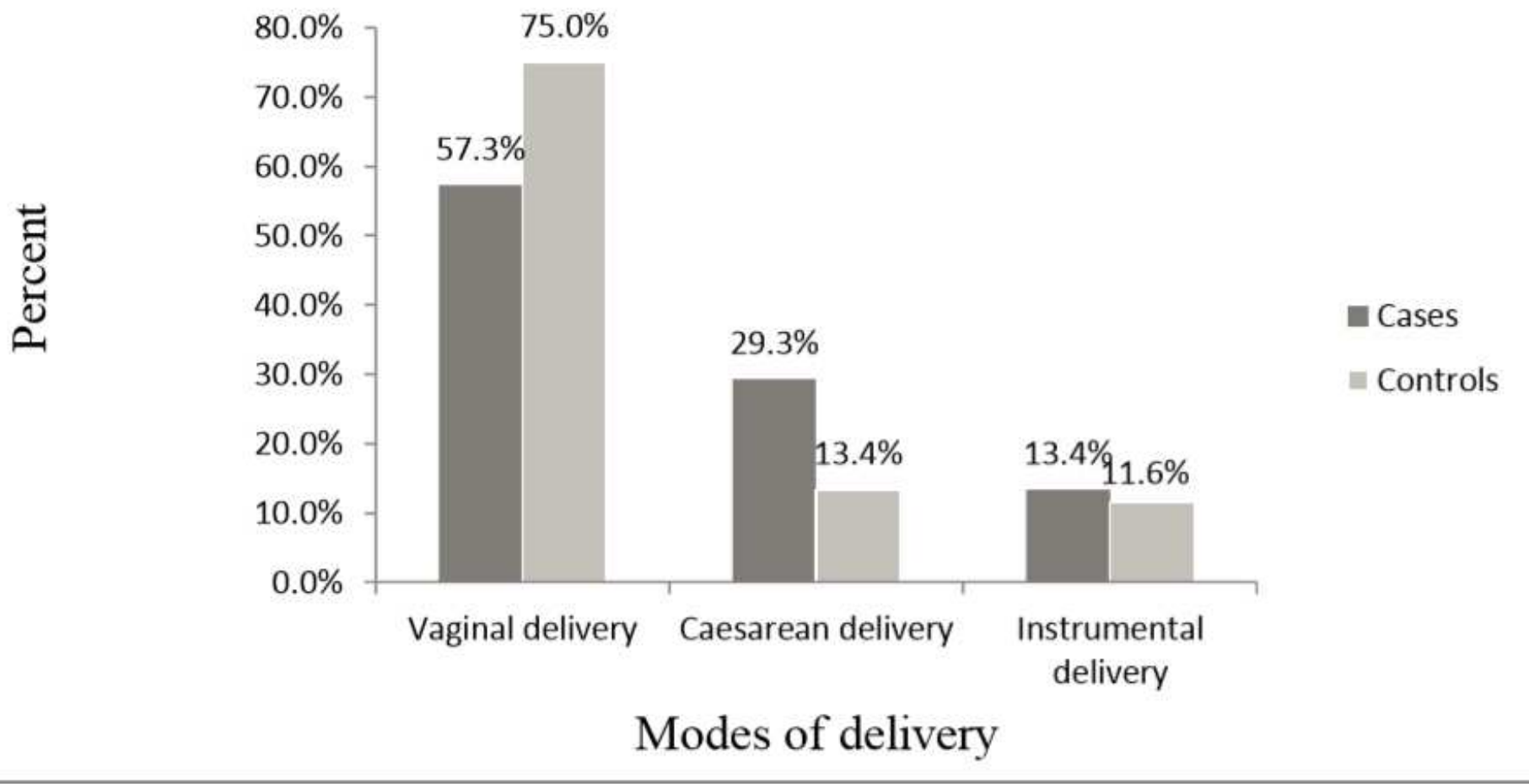

Figure 1

Mode of delivery in cases and controls for the study of determinants of necrotizing enterocolitis among neonates admitted in NICU at referral hospitals of East and West Gojjam Zones of Amhara regional state, Northwest Ethiopia, 


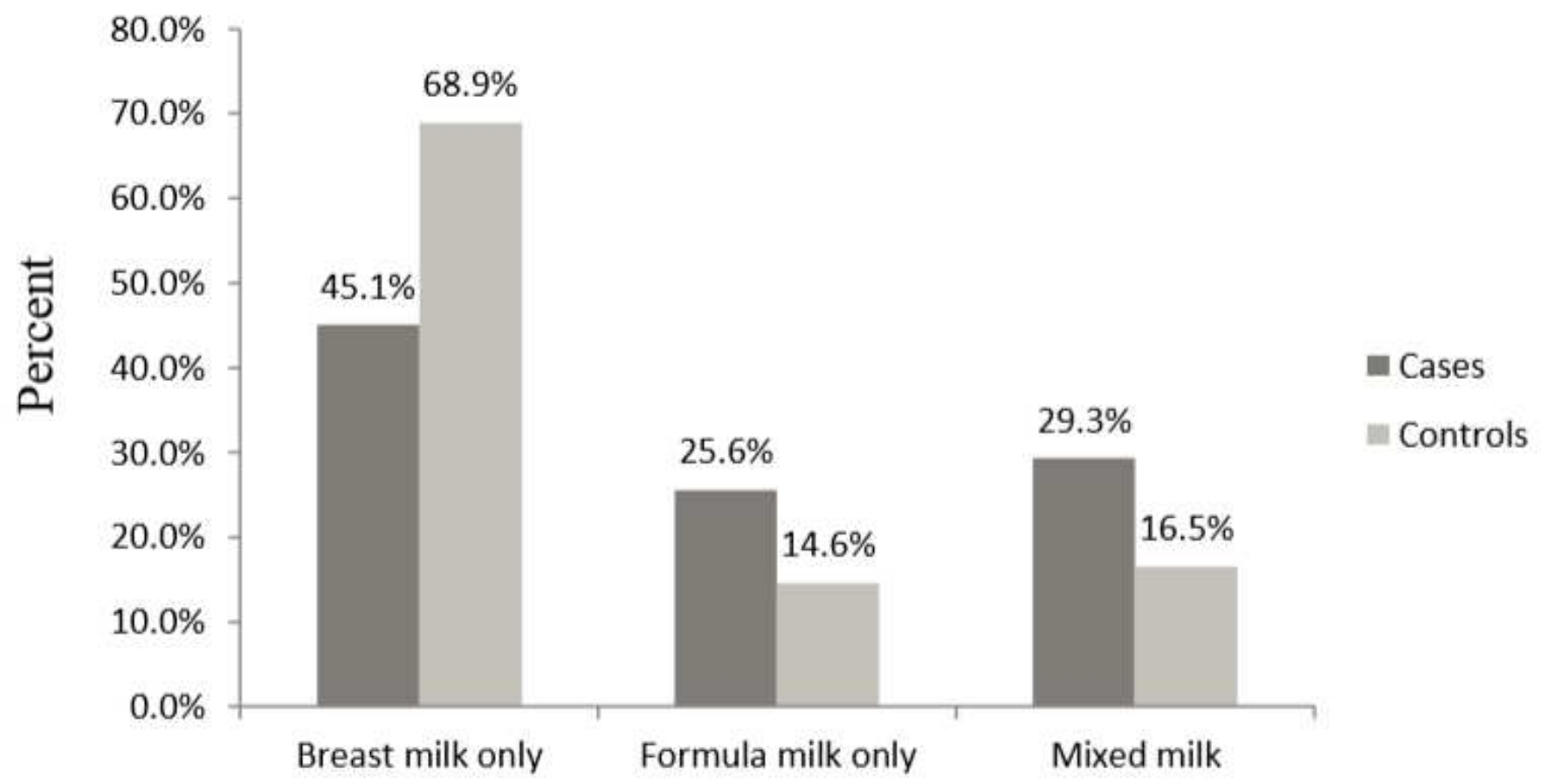

Types of enteral feeding

Figure 2

Types of enteral feeding for the study of determinants of necrotizing enterocolitis among neonates admitted in NICU at referral hospitals of East and West Gojjam Zones of Amhara regional state, Northwest Ethiopia, 2020. 